\title{
Modification of Urushiol Derivatives by Liquid Crystal Epoxy Resin
}

\author{
Gongwen Tang, Zhishan Yan, Linrong Ma, and Xin Huang \\ The Institute of Seawater Desalination and Multipurpose Utilization, SOA, Tianjin 300192, China \\ Correspondence should be addressed to Gongwen Tang; 13752134728@163.com
}

Received 2 December 2014; Revised 26 January 2015; Accepted 26 January 2015

Academic Editor: Rong Lu

Copyright ( 2015 Gongwen Tang et al. This is an open access article distributed under the Creative Commons Attribution License, which permits unrestricted use, distribution, and reproduction in any medium, provided the original work is properly cited.

\begin{abstract}
Urushiol derivatives have vast potentials for using as coating materials. However, the cured coatings are quite brittle, limiting their applications. In this study, urushiol-furfural (UFUR) was chosen as an example of urushiol derivatives and a liquid crystal (LC) epoxy resin, tetramethylbiphenyl diglycidyl ether (TMBPDE), was for the first time utilized to modify UFUR. Fourier transform infrared spectroscopy and solid-state ${ }^{13} \mathrm{C}$ nuclear magnetic resonance showed the reactions between TMBPDE and UFUR after the UFUR/TMBPDE composite resin was cured. Differential scanning calorimetry analysis showed that the $T_{g}$ significantly increased after the addition of TMBPDE. Thermogravimetry analysis indicated that the cured UFUR/TMBPDE composite resin exhibited increasing thermodecomposition temperature as the TMBPDE concentration increased, indicating its great potential for high temperature applications. Moreover, the presence of TMBPDE enhanced the toughness of UFUR as observed by impact test and reflected in the morphologies observed from SEM images of fracture surfaces. It would also be novel and effective to modify urushiol derivatives by the LC polymer.
\end{abstract}

\section{Introduction}

Oriental lacquer, known as the king of paints, has been used as coating materials for several thousand years in China [1]. It is a unique invention of uninterrupted civilization with 7000 years and becomes one of the most important sources of the chemistry in China [2]. Oriental lacquer is cut from phloem of the lacquer tree, an important economic tree of China [3]. The dried lacquer coatings possess a beautiful surface and high durability. Therefore, it has been used as decorative coatings to make life more beautiful and has become the symbol of wealth and status in ancient China $[2,4]$. Lacquer has been also widely used in chemical, textile, industry, ship-building, mechanical, and electrical products [5]. However, the limited production causes the high price of natural lacquer, which limits its application [6]. Lacquer paint dries by enzymatic oxidation of laccase and autoxidation of urushiol unsaturated side chains [7]. The curing progress is really slow and is strongly affected by the environmental conditions such as humidity and temperature [8]. Moreover, lacquer has poor flexibility, adhesion, alkali resistance, and ultraviolet degradation resistance. Therefore, modification of original lacquer is essential to expand its application fields [9]. Takahisa et al. [10] developed hybrid lacquers composed of natural lacquer and amine-functionalized organic silane compounds that showed good drying property at low relative humidity. Kanehashi et al. [11] reported hybrid microwaveadsorption materials prepared from natural lacquer, epoxy, and organic silane compounds. The results suggested that the chemical reactions among natural lacquer, epoxy, and organic silane improved the hybrid properties such as drying, hardness, and molding.

In general, urushiol, which was the main component of lacquer, was extracted from lacquer and chemically modified to obtain the urushiol derivatives. The urushiol derivatives exhibited superior properties and were used in various fields. In 1960, urushiol formaldehyde resin was synthesized by condensation reaction of urushiol and formaldehyde, which was a significant progress for lacquer modification in China [12]. Zhang and He [13] used inorganic boron to modify the urushiol formaldehyde polymer. Compared with urushiol formaldehyde resin, urushiol organic boron product has a higher antioxidant capacity, heat resistance, and transient high temperature performance. In addition, 
Liu [14] synthesized urushiol-furfural resin (UFUR) which could be formulated to a varnish with excellent abrasionresistance. However, the varnish was very brittle. Therefore, they modified the UFUR by epoxy E-12. The modified varnish showed excellent mechanical properties, especially the abrasion-resistance, corrosion protection, and alkali resistance. UFUR could be cured by heating and it own some superior properties. Therefore, it is quite necessary to find new ways to modify UFUR.

Liquid crystal (LC) epoxy resins have been investigated because of their unique properties, for example, low shrinkage upon curing, good thermal stability, and excellent thermomechanical properties [15]. They combine the advantages of both LCs and epoxy resins. Compared to ordinary epoxy resins, crosslinked LC epoxy resins have higher fracture toughness [16]. Traditional epoxy resins can be improved by introducing LC domains into the amorphous matrix [17]. Unlike other toughening methods such as incorporating rubber particles, the presence of LC domains will not lead to a decrease in the glass transition temperature $\left(T_{q}\right)$ or moduli of the material. These desirable properties make LC epoxy resins good candidates for a wide range of potential applications, such as optical switches, electronic packaging, and matrices for high performance composites [15]. Many efforts were directed towards the increase of toughness by introduction of LC epoxy resins in epoxy resins [18-21]. For example, Punchaipetch et al. $[18,19]$ copolymerized diglycidyl ether of 4,4-dihydroxybiphenol (DGE-DHBP) with diglycidyl ether of bisphenol $\mathrm{F}$ networks and cured it with an anhydride curing agent as the matrix resins. DGE-DHBP enhances the toughness of the blended epoxy samples without decreasing the modulus of samples. However, to the best of our knowledge there are few researches on modification of UFUR with LC polymers.

In the current study, UFUR was chosen as an example of urushiol derivatives and an effective modification method was proposed. A LC epoxy resin, tetramethylbiphenyl diglycidyl ether (TMBPDE), was utilized to modify UFUR for the first time. The LC property of TMBPDE was investigated by polarized optical microscope (POM). The effects of TMBPDE on the $T_{g}$, thermal stability, and impact strength of the UFUR/TMBPDE composite resin were analyzed. The fracture surfaces of UFUR/TMBPDE composite resins were investigated by scanning electron microscope (SEM).

\section{Materials and Methods}

2.1. Materials. TMBPDE was supplied from Gansu Research Institute of Chemical Industry with an epoxy equivalent weight of 192. UFUR was purchased from Wuhan GuoQi Co., Ltd., China. Dimethylbenzene, acetone, and other chemicals were supplied by Tianjin Kemiou Chemical Reagent Co., Ltd., China, and used as received. The chemical structures of TMBPDE and UFUR are shown in Figure 1.

2.2. Sample Preparation. A certain amount of TMBPDE was dissolved in acetone to obtain a solution with the concentration of $25 \mathrm{wt} \%$. Dimethylbenzene solution of UFUR
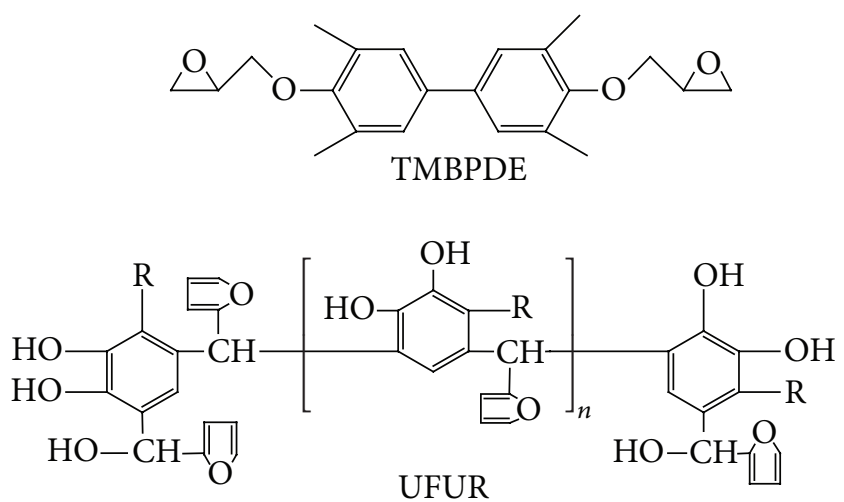

Figure 1: The chemical structures of TMBPDE and UFUR.

was taken in a beaker. Then, a given ratio of TMBPDE was added and mixed for $10 \mathrm{~min}$ at room temperature. The mixture was placed in fume hood to volatilize most solvents at room temperature. For preparation of samples for impact strength test, the above mixture was heated at $80^{\circ} \mathrm{C}$ for $0.5 \mathrm{~h}$ and immediately poured into a preheated mold (inside size: $80 \mathrm{~mm} \times 10 \mathrm{~mm} \times 4 \mathrm{~mm}$ ) at room temperature. The samples in the molds were cured at $80^{\circ} \mathrm{C}$ for $4 \mathrm{~h}, 110^{\circ} \mathrm{C}$ for $4 \mathrm{~h}$, and $160^{\circ} \mathrm{C}$ for $4 \mathrm{~h}$ in an air-circulating oven. All samples prepared for other characterizations were cured as follows. The mixture was coated onto the treated galvanized iron sheets, dried at room temperature for $1-3 \mathrm{~h}$, and cured at $110^{\circ} \mathrm{C}$ for $2 \mathrm{~h}$ and $160^{\circ} \mathrm{C}$ for $2 \mathrm{~h}$ in an air-circulating oven.

2.3. Characterization and Test. In order to study the effects of TMBPDE on the properties of UFUR, different contents of TMBPDE were added. Samples with the TMBPDE contents of $0 \%, 5 \%, 15 \%$, and $30 \%$ with respect to UFUR were marked as TMBPDE-0, TMBPDE-5, TMBPDE-15, and TMBPDE-30, respectively.

LC property of TMBPDE was investigated using a polarized optical microscope (POM) from Leica (model DM 4500P equipped with a Linkam LTS-350 hot stage and TMS94 temperature controller). A small amount of TMBPDE was placed on a microscope slide and then covered with a piece of cover glass. The formation and development of the LC phase were examined under polarized light. The sample was heated and cooled repeatedly from room temperature to $140^{\circ} \mathrm{C}$ at a rate of $10^{\circ} \mathrm{C} / \mathrm{min}$ to investigate the change of birefringence.

The structure analyses of UFUR/TMBPDE composite resin upon curing were conducted via FTIR spectra and solid-state nuclear magnetic resonance (NMR) spectra. FTIR spectra was recorded in a spectrum 100 FTIR spectrometer (FTS3000, BIORAD, USA) in the range from $4000 \mathrm{~cm}^{-1}$ to $400 \mathrm{~cm}^{-1}$. A potassium bromide pellet was used to support the IR sample. The sample was spread thinly on the potassium bromide pellet with a spatula, and the potassium bromide pellet was used as a blank. The solid-state ${ }^{13} \mathrm{C}$ NMR spectra were recorded on a JNM-ECA400 (JEOL Co. Ltd., $100 \mathrm{MHz}$ ) using zirconium sample tubes with the CP/MAS method.

The effects of TMBPDE on the $T_{g}$ and thermal stability of the composite resin were investigated by differential scanning 


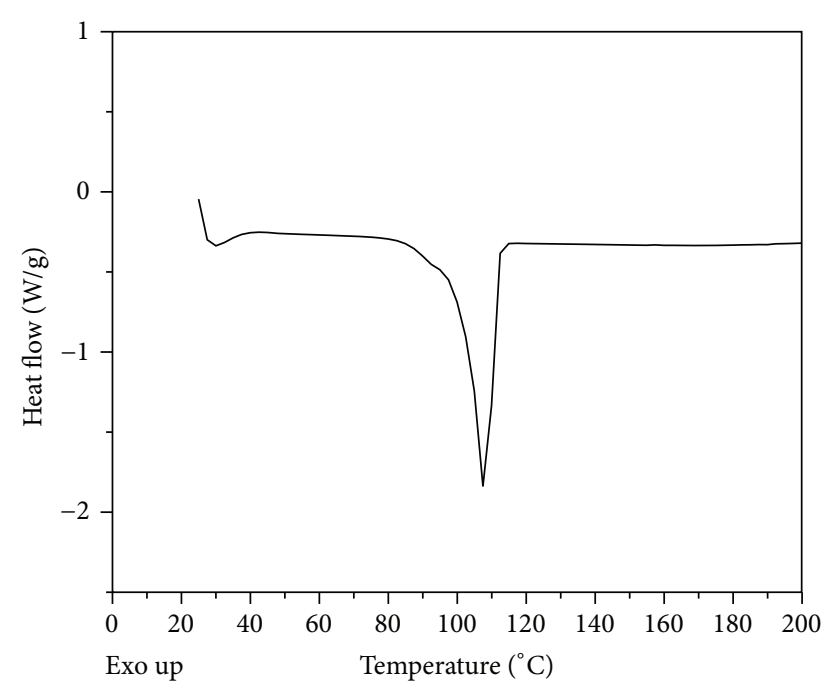

FIGURE 2: DSC thermogram of TMBPDE.

calorimetry (DSC) and thermogravimetry (TG) analysis with a simultaneous thermal analysis (DSC/DTA-TG, NETZSCH, STA 409 PC Luxx, Germany) under the nitrogen atmosphere at a heating rate of $10^{\circ} \mathrm{C} / \mathrm{min}$ from $30^{\circ} \mathrm{C}$ to $500^{\circ} \mathrm{C}$.

Impact strength was measured by an impact testing machine according to the GB/T1843-2008 standard. The samples were tested by the cantilever beam impact testing machine (Chengde Precision Tester XC-22, China). The sample was placed into the sample well and the pendulum bob fell through a vertical height on the surface. The morphologies of the fracture surfaces after impact tests were coated with gold and observed using SEM (Philips XL30, Dutch).

\section{Results and Discussion}

3.1. The UFUR/TMBPDE Composite Resin. It was generally believed that the ordered structure that was formed during cured process improved the toughness of the modified resins. If the composite resin was cured during the LC temperature range, more ordered mesogenic domains would be formed. Therefore, it is necessary to determine the LC temperature range. To investigate mesomorphic phase transition of TMBPDE, DSC and POM studies were carried out. The DSC thermogram of TMBPDE is shown in Figure 2. Only one endothermic peak was observed at about $107^{\circ} \mathrm{C}$ in the heating scan, which was the melting of TMBPDE. However, the clearing point of LC was not found. The thermal behavior of some liquid crystal monomer is not well understood. For example, Liu et al. [22] found two phase transition peaks in the heating scan of DSC. Li and coworkers [15] were not able to detect any LC phase of $4,4^{\prime}$-diglycidyloxybiphenyl (BP) upon heating or observed a LC phase by POM. However, upon reacting with sulfanilamide, a smectic LC phase started forming after $20 \mathrm{~min}$ of the curing reaction. The TMBPDE had similar structure as BP. We were also not able to detect any LC phase of TMBPDE upon heating by DSC.

As we know, POM is a powerful tool for characterization of liquid phases. In order to clarify the mesophases of TMBPDE under POM, the samples were prepared by sandwiching a tiny power between two glass plates. Figure 3 shows the POM images of TMBPDE taken at different temperatures (200x magnification). TMBPDE started to melt at $107^{\circ} \mathrm{C}$, which is in good agreement with the DSC data. At the same time, LC birefringence was formed, which presents highly birefringent and nematic liquid crystal behavior. The texture color changed with increasing temperature. When the temperature was higher than $117^{\circ} \mathrm{C}$, the $\mathrm{POM}$ image turned to be completely dark due to the disappearance of the LC phase.

In order to examine the reaction between UFUR and TMBPDE in the composite resin during the curing process, the FTIR spectrums of UFUR and UFUR with 15\% TMBPDE before and after being cured were carried out. The results are shown in Figure 4. After the addition of TMBPDE, the peak at $910 \mathrm{~cm}^{-1}$ due to the absorption of the epoxy group appeared. After the UFUR/TMBPDE composite resin was cured, the peak at $910 \mathrm{~cm}^{-1}$ disappeared and the peak at $3400 \mathrm{~cm}^{-1}$ due to the hydroxyl groups decreased, suggesting that polymerization between the epoxy group and the hydroxyl groups had occurred. A decrease in the hydroxyl group peak also promoted the autoxidation of UFUR.

The solid-state ${ }^{13} \mathrm{C}$ NMR spectra of TMBPDE, UFUR, and cured TMBPDE/UFUR composite resin are shown in Figure 5. The peak at ca. $17 \mathrm{ppm}$ belonging to carbon of methyl on the urushiol aromatic ring of TMBPDE was detected in the spectrum of TMBPDE/UFUR composite resin. For TMBPDE, the oxygen atom substituted carbons on the epoxy group were detected at ca. $45 \mathrm{ppm}$ and $52 \mathrm{ppm}$ (Figure 5(a)). After the TMBPDE/UFUR composite resin was cured, the peaks of these carbons disappeared (Figure 5(c)), indicating that polymerization between TMBPDE and UFUR had occurred during curing.

In conclusion, although the LC phase of TMBPDE could not be detected by DSC, it could be observed by POM. The LC temperature range was from $107^{\circ} \mathrm{C}$ to $117^{\circ} \mathrm{C}$. During curing, UFUR/TMBPDE composite resin was cured at $110^{\circ} \mathrm{C}$ for $2 \mathrm{~h}$ and then $160^{\circ} \mathrm{C}$ for $2 \mathrm{~h}$. At $110^{\circ} \mathrm{C}$, TMBPDE presented a LC phase and it had enough time for the mesogenic segments to form LC domains during the curing process [15]. Figure 6 shows POM images of UFUR/TMBPDE composite resin taken at different temperatures (100x). The LC phase starts to appear above $100^{\circ} \mathrm{C}$. As the temperature increased, the LC phase did not disappear. After being further cured at $160^{\circ} \mathrm{C}$ for $2 \mathrm{~h}$, the LC domains would be "locked" in the network of the composite resin.

3.2. Thermal Properties. The cured thermosets are in glass state at room temperature. When the temperature was above its $T_{g}$, the uncured molecules could move in the microBrownian motion, which decreased the rigidity and strength. Therefore, the $T_{g}$ is an important index to measure the heat resistance and available range of thermosetting resins. Therefore, it is necessary to study the effect of TMBPDE on the $T_{g}$ of the composite resins. The relationship between the $T_{g}$ and the TMBPDE content of the composite sample was valuated by DSC. As shown in Figure $7, T_{g}$ of UFUR was about $187.2^{\circ} \mathrm{C}$. For UFUR/TMBPDE composite resins, 


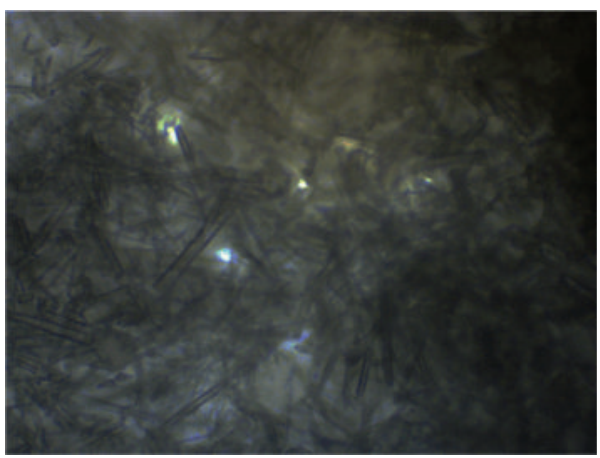

(a)

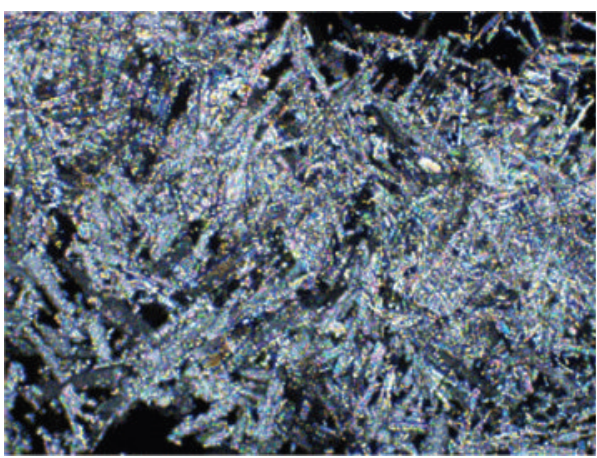

(c)

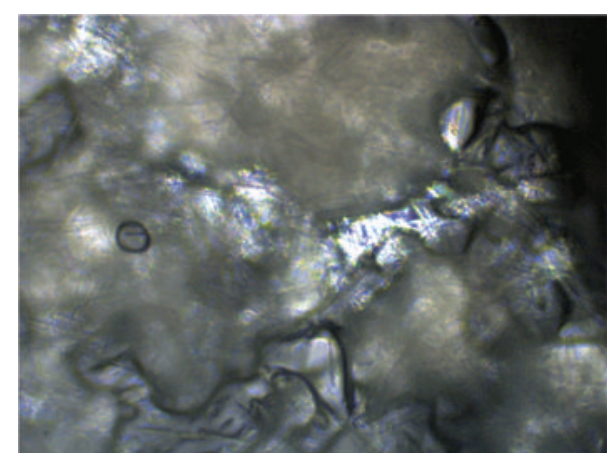

(b)

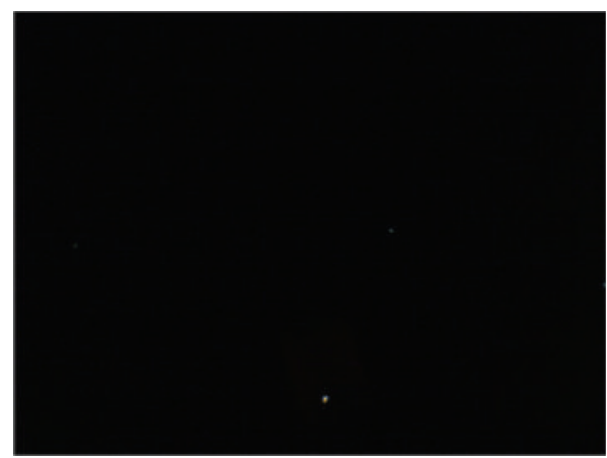

(d)

Figure 3: POM images of TMBPDE at different temperatures (200x): (a) $100^{\circ} \mathrm{C}$; (b) $107^{\circ} \mathrm{C}$; (c) $110^{\circ} \mathrm{C}$; (d) $118^{\circ} \mathrm{C}$.

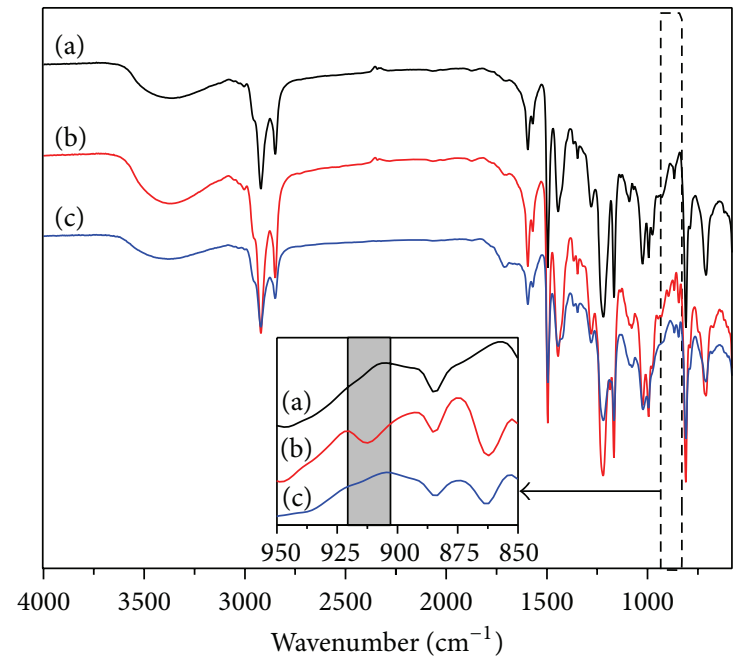

FIGURE 4: FTIR spectra of samples ((a) UFUR; (b) TMBPDE-15 (uncured); (c) TMBPDE-15 (cured)).

when TMBPDE content was less than $15 \%, T_{g}$ of the composite resin increased as the TMBPDE content increased. When $15 \%$ TMBPDE was added, the $T_{g}$ of the composite resin increased from $187.2^{\circ} \mathrm{C}$ to $232^{\circ} \mathrm{C}$. However, when the TMBPDE content reached $30 \%$, the $T_{g}$ of the composite resin decreased to $224.1^{\circ} \mathrm{C}$. Huang et al. [23] found that the

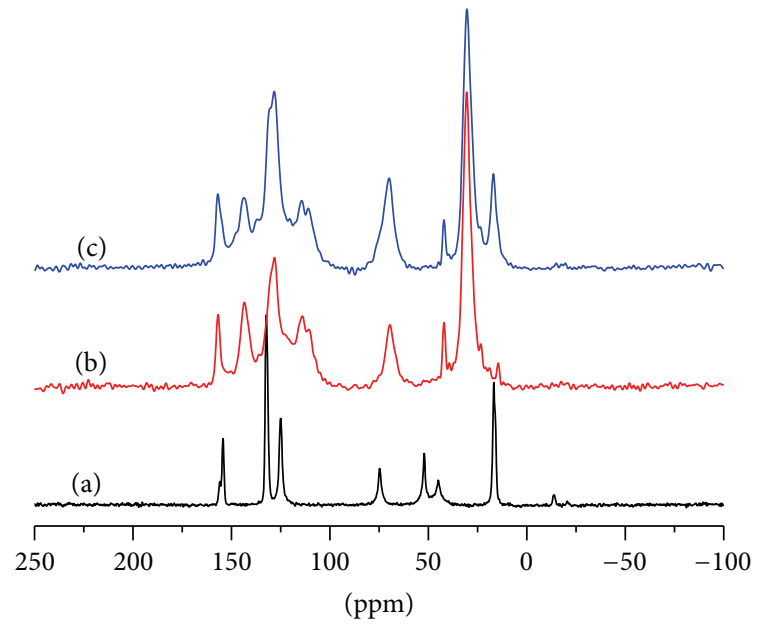

Figure 5: ${ }^{13} \mathrm{C}$ CP/MAS NMR spectra of samples ((a) TMBPDE; (b) UFUR; (c) TMBPDE-15 (cured)).

novel liquid crystalline polymers, polydiethyleneglycol bis(4hydroxybenzoate) terephthaloyl and block copolymer, could enhance the $T_{g}$ of the o-cresol formaldehyde epoxy resin remarkably due to the rigid segments in the liquid crystalline polymers. Therefore, in this study, it is also proposed that the rigid segments in TMBPDE are the main reason why TMBPDE can improve $T_{g}$ of the composite resins. However, when the TMBPDE content was improved to $30 \%, T_{g}$ was 


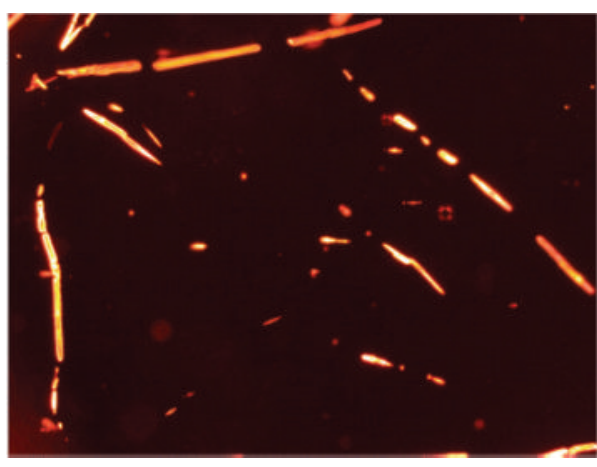

(a)

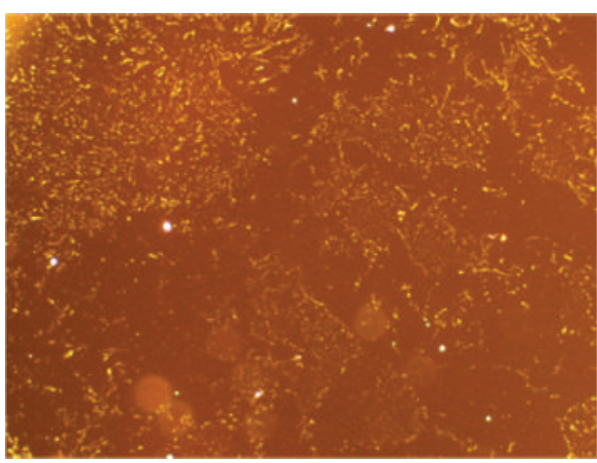

(c)

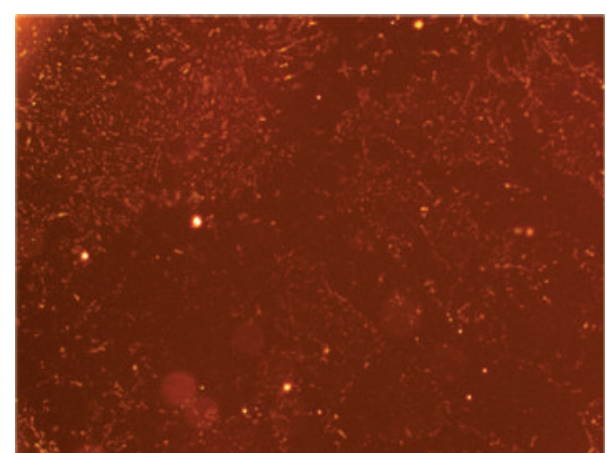

(b)

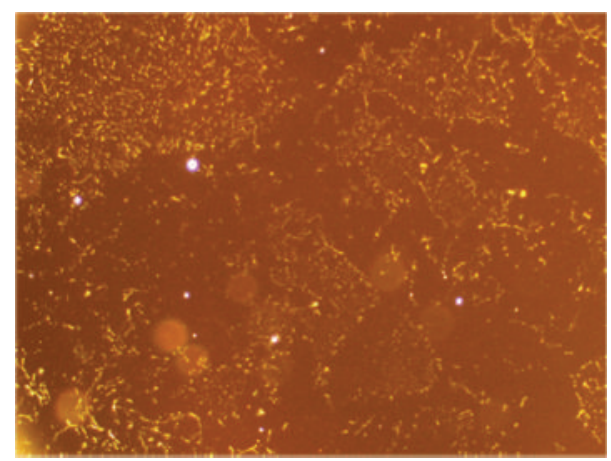

(d)

FIGURE 6: POM images of UFUR/TMBPDE composite resin at different temperatures (100x): (a) $90^{\circ} \mathrm{C}$; (b) $100^{\circ} \mathrm{C}$; (c) $110^{\circ} \mathrm{C}$; (d) $160^{\circ} \mathrm{C}$.

TABLE 1: Thermodata obtained from DSC $\left(T_{g}\right)$ and TG $\left(T_{d}\right)$

\begin{tabular}{lcc}
\hline Remarks & $T_{g}\left({ }^{\circ} \mathrm{C}\right)$ & $T_{d}\left({ }^{\circ} \mathrm{C}\right)$ \\
\hline TMBPDE-0 & 187 & 330 \\
TMBPDE-5 & 195 & 335 \\
TMBPDE-15 & 232 & 345 \\
TMBPDE-30 & 224 & 352 \\
\hline
\end{tabular}

lower than the former but much higher than that of UFUR. We found similar phenomenon to Huang et al. [23], who found that $T_{g}$ of the blends first increased and then decreased as the content of the liquid crystalline polymers is increasing. It was proposed that the molecular weight of TMBPDE was small. Therefore, when $30 \mathrm{wt} \%$ of TMBPDE was added, the structure density of the curing network would decrease, leading to the slight decrease of $T_{g}$ of TMBPDE-30. However, this problem will need to be further gone into.

Effects of TMBPDE on thermal stability of the composite resins were also investigated. Figure 8 shows the TGA curves for UFUR/TMBPDE composite resins with different TMBPDE contents. The thermal degradation temperature $\left(T_{d}\right)$ was defined as the temperature when the samples lost $5 \%$ of their initial weight, and the results were summarized in Table 1. It can be seen that the $T_{d}$ s of all UFUR/TMBPDE composite resins were higher than that of pure UFUR, indicating that the addition of TMBPDE enhanced thermal stability of UFUR. For the composite resins, the $T_{d}$ s increased with the TMBPDE contents. When the content of TMBPDE was $15 \%$, the $T_{d}$ of the composite resin increased to $352^{\circ} \mathrm{C}$. The high value of the decomposition temperature can be ascribed to the presence of the biphenyl as rigid block. Therefore, TMBPDE strongly enhances the thermal stability of the UFUR/TMBPDE composite resin.

3.3. Impact Strength. Figure 9 shows the comparison of impact strength as a function of the TMBPDE contents. There was an increase in the impact strength of the composite resin on addition of the TMBPDE. When 30\% TMBPDE was added, the UFUR/TMBPDE composite resin showed a fourfold increase in impact strength compared to pure UFUR. Punchaipetch [19] also has reported that the incorporation of a relatively small amount of the LC epoxy (diglycidyl ether of $4,4^{\prime}$-dihydroxybiphenol) could significantly enhance the toughness and $T_{g}$ of the blended epoxy samples.

The impact surfaces were investigated by SEM, as shown in Figure 10. The cured UFUR exhibited a brittle type of fracture behavior. For the cured composite resin with 5\% TMBPDE, the fracture surface was very sharp with no plastic deformation. The crack grew in one direction because of the minimum resistance. When the addition of TMBPDE was increased to $15 \%$, the rigid segments which were crosslinked in the UFUR matrix could absorb some impact energy, 


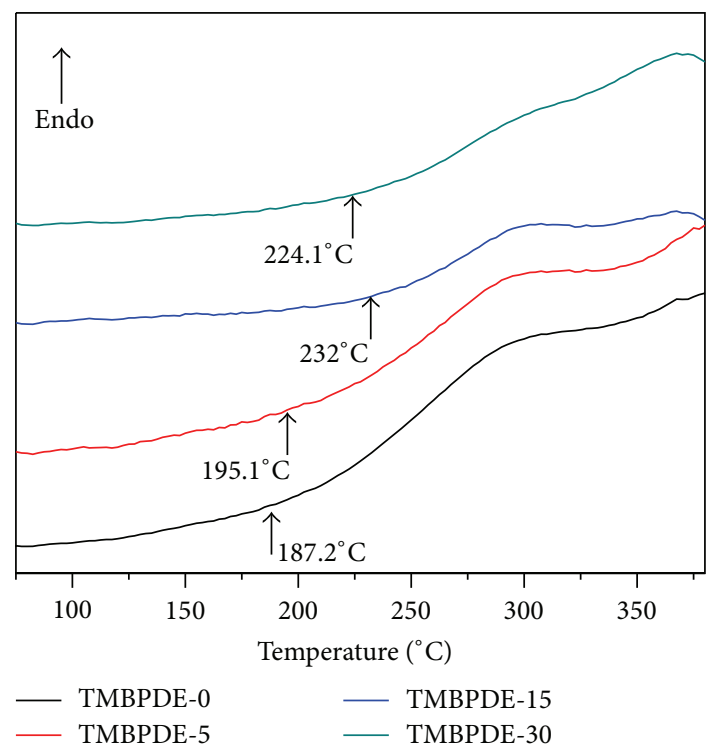

Figure 7: The glass transition temperatures of UFUR/TMBPDE composite resins with different TMBPDE contents.

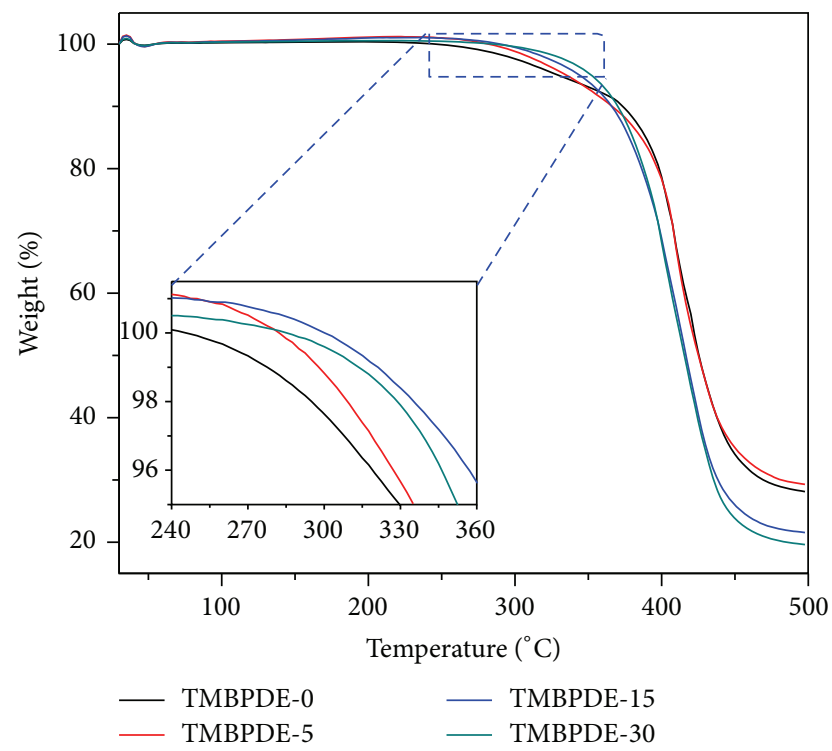

FIGURE 8: Thermogravimetric analysis of UFUR/TMBPDE composite resins with different TMBPDE contents.

resulting in improvement of the toughness. Therefore, the fracture surface exhibited ductile fracture with a river pattern where the multiple lines begin as a single line at the initiation point. For the composite resin with 30\% TMBPDE, the furrow pattern at the starter crack region is found to be much bigger and coarser than that with 15\% TMBPDE. Therefore, the toughness of TMBPDE-30 was higher than TMBPDE-15 by SEM.

The LC possesses a unique hierarchical microstructure in which, in the absence of external fields, they tend to

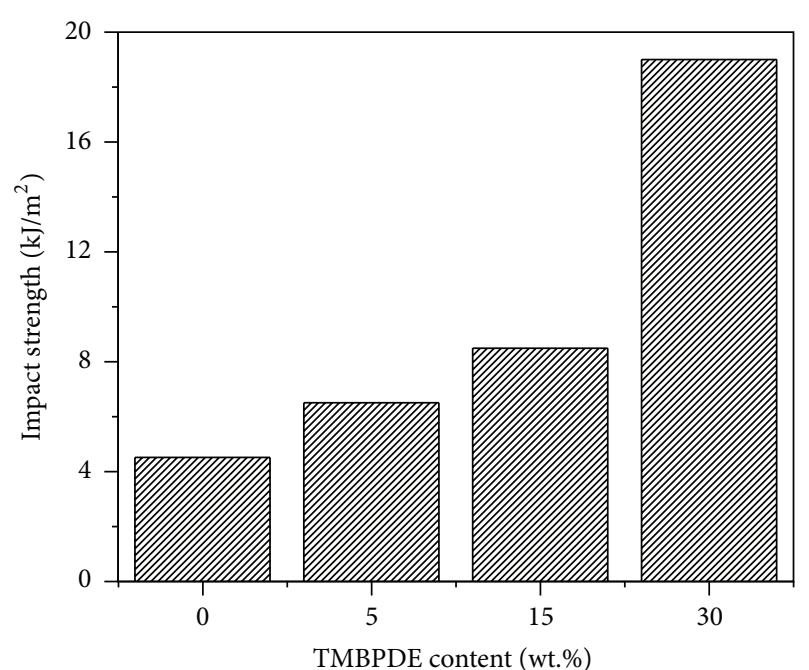

FIGURE 9: Impact strength of UFUR/TMBPDE composite resins with different TMBPDE contents.

form a macroscopically disordered polydomain, where each LC domain is defined as a region of uniform orientation [24]. For the LC thermoset, it exhibits exceptionally high fracture toughness. At low stress, crazes first take place within the isotropic amorphous domains and grow along the stress direction. Finally the crazes are hampered by the LC domains, which improved the impact strength of the LC thermoset [25]. For the UFUR/TMBPDE composite resin in our study, during curing, the LC phase of TMBPDE was "frozen" into the network, forming anisotropic LC domains surrounded by a macroscopically disordered polydomain. Therefore, the microdeformation and fracture mechanism of the LC thermoset could be extended to the case of the UFUR/TMBPDE networks and explain the reason for the impact strength improvement of the composite resin.

\section{Conclusions}

TMBPDE was utilized to modify the urushiol derivative, UFUR. The results showed that the $T_{g}$ significantly increased after the addition of TMBPDE. The UFUR/TMBPDE composite resin with $15 \%$ TMBPDE had a higher $T_{g}$ by $45^{\circ} \mathrm{C}$ compared with the pure UFUR. TGA analysis showed that the cured UFUR/TMBPDE composite resin exhibited increasing thermodecomposition temperature as the TMBPDE concentration increased, indicating its great potential for high temperature applications. Moreover, the presence of TMBPDE enhanced the toughness of UFUR as observed by impact test and reflected in the morphologies observed from SEM images of fracture surfaces. In conclusion, TMBPDE significantly enhanced the toughness of UFUR without sacrificing high $T_{g}$ and thermodecomposition temperature. It is supposed that the improvements of the properties are the effects of the mesogenic domains distributed in the network. These results also suggest that it would be a novel and effective way to modify other urushiol derivatives by a LC polymer. 


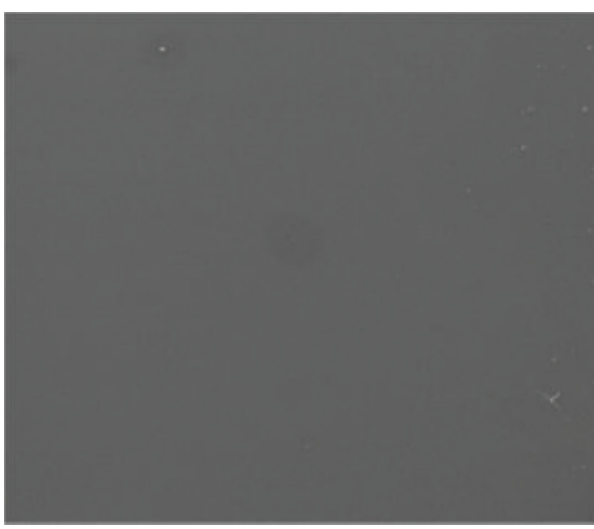

(a)

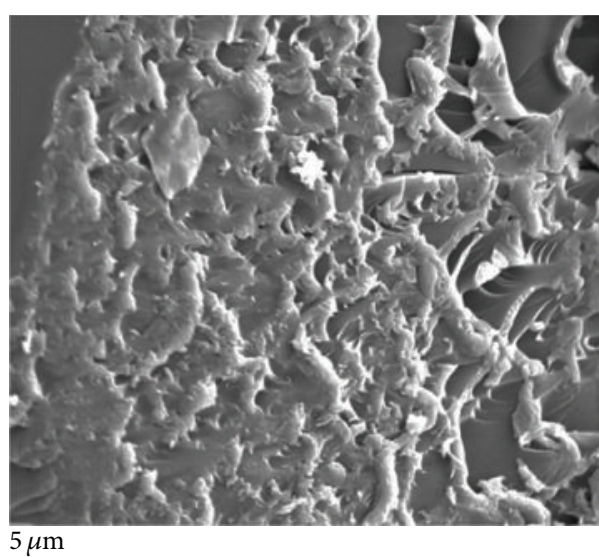

(c)

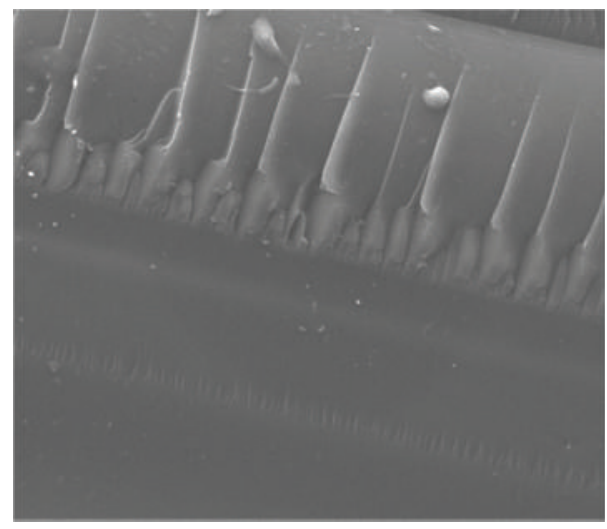

(b)

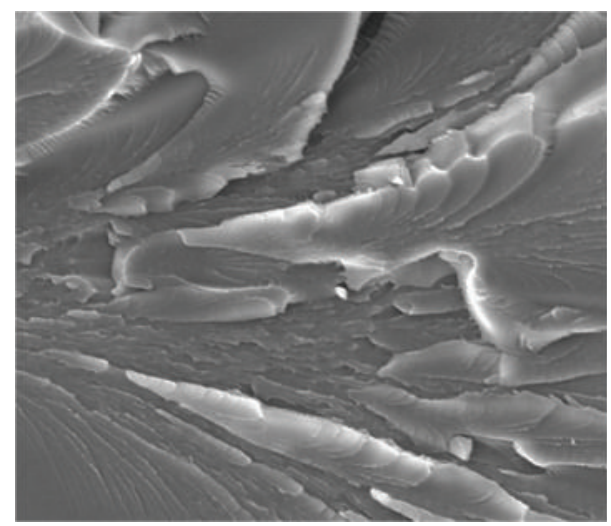

$5 \mu \mathrm{m}$

(d)

FIGURE 10: Impact surfaces of UFUR/TMBPDE composite resins ((a) TMBPDE-0; (b) TMBPDE-5; (c) TMBPDE-15; (d) TMBPDE-30).

\section{Conflict of Interests}

The authors declare that there is no conflict of interests regarding the publication of this paper.

\section{Acknowledgments}

This work was supported by Natural Science and Technology Ministry of China (ID: 2011BAD33B03) and the Key Technologies R\&D Program of Tianjin, China (ID: 14ZXCXGX00680).

\section{References}

[1] X. L. Sun, G. M. Wu, and Z. W. Kong, "Research progress of modification and application of raw lacquer," Biomass Chemical Engineering, vol. 48, no. 2, pp. 41-46, 2014.

[2] F. L. Zhang, "Origin of lacquer civilization in China," Journal of Chinese Lacquer, vol. 29, no. 2, pp. 16-25, 2010.

[3] D. X. Li and F. L. Zhang, "Extraction of active constituents and progress in the studies on Lacquer tree," Journal of Chinese Lacquer, vol. 33, no. 1, pp. 27-32, 2014.

[4] F. L. Zhang and H. Zhang, "Lacquer tapping and refined processing technology in ancient China," Journal of Chinese Lacquer, vol. 30, no. 2, pp. 22-27, 2011.
[5] R. Lu, N. Ebata, F.-L. Zhang, and T. Miyakoshi, "Development of a new type lacquer based on Rhus vernicifera sap with chitosan," Progress in Organic Coatings, vol. 77, no. 2, pp. 439-443, 2014.

[6] J. Kumanotani, "Urushi (oriental lacquer)-a natural aesthetic durable and future-promising coating," Progress in Organic Coatings, vol. 26, no. 2-4, pp. 163-195, 1995.

[7] F. L. Zhang, "Molecular mechanism of lacquer film formation," Journal of Chinese Lacquer, vol. 31, no. 1, pp. 13-20, 2012.

[8] T. Ishimura, R. Lu, K. Yamasaki, and T. Miyakoshi, "Effects of hybridization of lacquer sap with organic silane on drying properties," Progress in Organic Coatings, vol. 62, no. 2, pp. 193198, 2008.

[9] Q. Wang, Y. Shi, and F. L. Zhang, "Development progress on the modification of natural raw lacquer," Shanghai Coatings, vol. 48, no. 2, pp. 30-33, 2010.

[10] I. Takahisa, L. Rong, and M. Tetsuo, "Studies on the reaction mechanism between urushiol and organic silane," Progress in Organic Coatings, vol. 55, no. 1, pp. 66-69, 2006.

[11] S. Kanehashi, H. Oyagi, R. Lu, and T. Miyakoshi, "Development of bio-based hybrid resin, from natural lacquer," Progress in Organic Coatings, vol. 77, no. 1, pp. 24-29, 2014.

[12] B. $\mathrm{Hu}, \mathrm{J}$. Lin, and $\mathrm{Y} . \mathrm{Xu}$, "The improvement of raw lacquer and the study on urushiol-metal polymer," Journal of Chinese Lacquer, vol. 19, no. 3, pp. 1-7, 2000. 
[13] F. L. Zhang and N. He, "Synthesis and characterization of urushiol organic boron resin," Shanghai Coatings, vol. 31, no. 2, pp. 3-5, 2012.

[14] Y. Liu, "Epoxy modified urushiol-furfural resin," Paint \& Coatings Industry, vol. 3, pp. 1-4, 1995.

[15] Y. Li, P. Badrinarayanan, and M. R. Kessler, "Liquid crystalline epoxy resin based on biphenyl mesogen: thermal characterization," Polymer, vol. 54, no. 12, pp. 3017-3025, 2013.

[16] C. Carfagna, E. Amendola, and M. Giamberini, "Liquid crystalline epoxy based thermosetting polymers," Progress in Polymer Science, vol. 22, no. 8, pp. 1607-1647, 1997.

[17] M. Harada, N. Okamoto, and M. Ochi, "Fracture toughness and fracture mechanism of liquid-crystalline epoxy resins with different polydomain structures," Journal of Polymer Science, Part B: Polymer Physics, vol. 48, no. 22, pp. 2337-2345, 2010.

[18] P. Punchaipetch, V. Ambrogi, M. Giamberini, W. Brostow, C. Carfagna, and N. A. D’Souza, "Epoxy + liquid crystalline epoxy coreacted networks: I. Synthesis and curing kinetics," Polymer, vol. 42, no. 5, pp. 2067-2075, 2001.

[19] P. Punchaipetch, V. Ambrogi, M. Giamberini, W. Brostow, C. Carfagna, and N. A. D’Souza, "Epoxy+liquid crystalline epoxy coreacted networks: II. Mechanical properties," Polymer, vol. 43, no. 3, pp. 839-848, 2002.

[20] H. J. Sue, J. D. Earls, and R. E. Hefner Jr., "Fracture behaviour of liquid crystal epoxy resin systems based on the diglycidyl ether of $4,4^{\prime}$-dihydroxy- $\alpha$-methylstilbene and sulphanilamide: part I Effects of curing variations," Journal of Materials Science, vol. 32, no. 15, pp. 4031-4037, 1997.

[21] P. Chang, R. Zuo, R. Wang, and L. Chen, “Toughening of epoxy resins with a liquid-crystalline epoxy resin," Acta Polymerica Sinica, no. 5, pp. 682-684, 2002.

[22] Y.-L. Liu, Z.-Q. Cai, X. Wen et al., “Thermal properties and cure kinetics of a liquid crystalline epoxy resin with biphenylaromatic ester mesogen," Thermochimica Acta, vol. 513, no. 1-2, pp. 88-93, 2011.

[23] Z. F. Huang, S. T. Tan, and X. Y. Wang, "Modification of an o-cresol formaldehyde epoxy resin by the thermotropic liquid crystalline polymer," Journal of Applied Polymer Science, vol. 97, no. 4, pp. 1626-1631, 2005.

[24] C. Ortiz, R. Kim, E. Rodighiero, C. K. Ober, and E. J. Kramer, "Deformation of a polydomain, liquid crystalline epoxy-based thermoset," Macromolecules, vol. 31, no. 13, pp. 4074-4088, 1998.

[25] C. Ortiz, L. Belenky, C. K. Ober, and E. J. Kramer, "Microdeformation of a polydomain, smectic liquid crystalline thermoset," Journal of Materials Science, vol. 35, no. 8, pp. 2079-2086, 2000. 

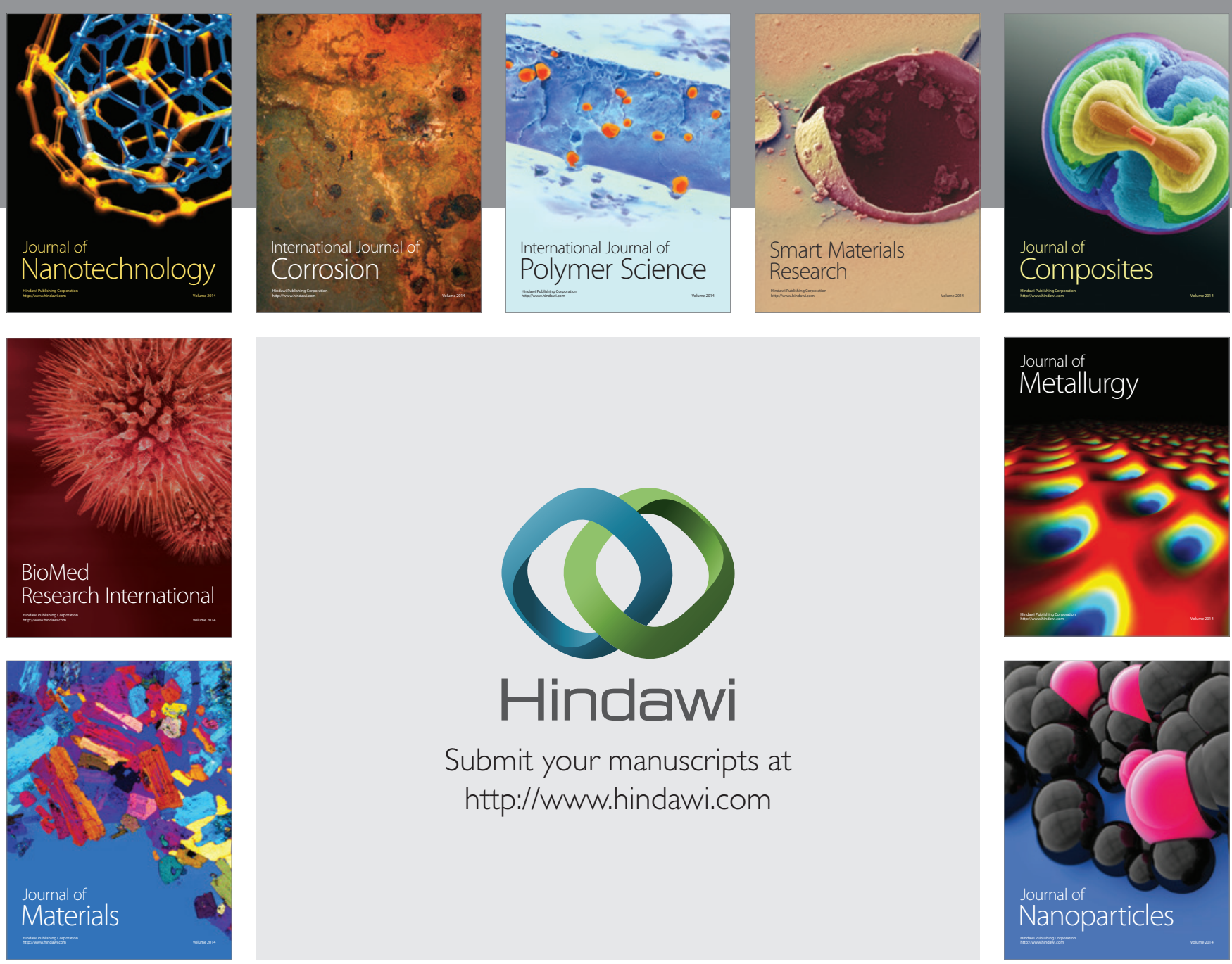

Submit your manuscripts at http://www.hindawi.com
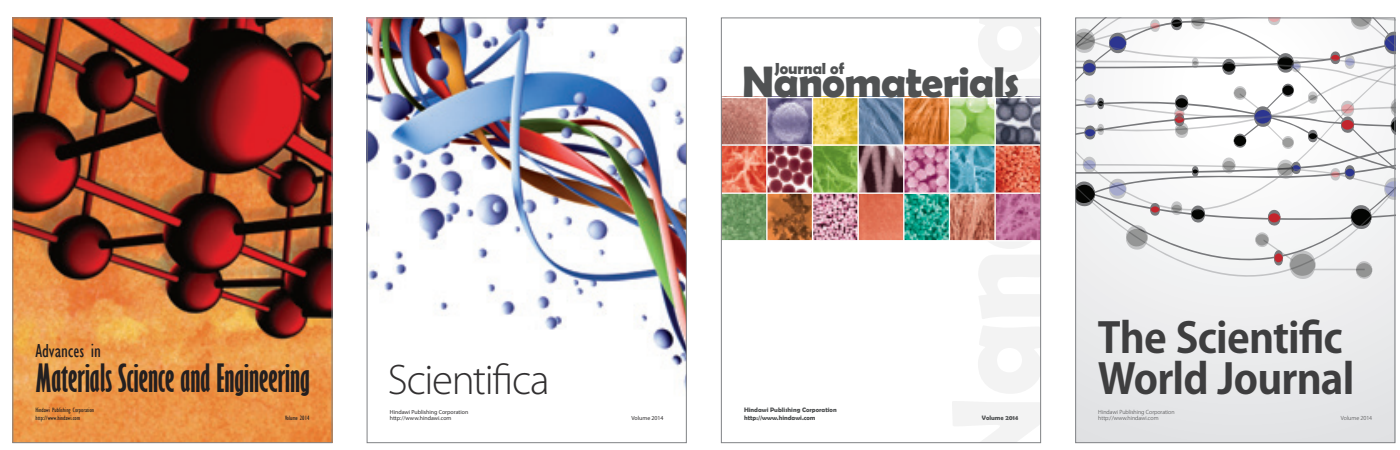

\section{The Scientific World Journal}
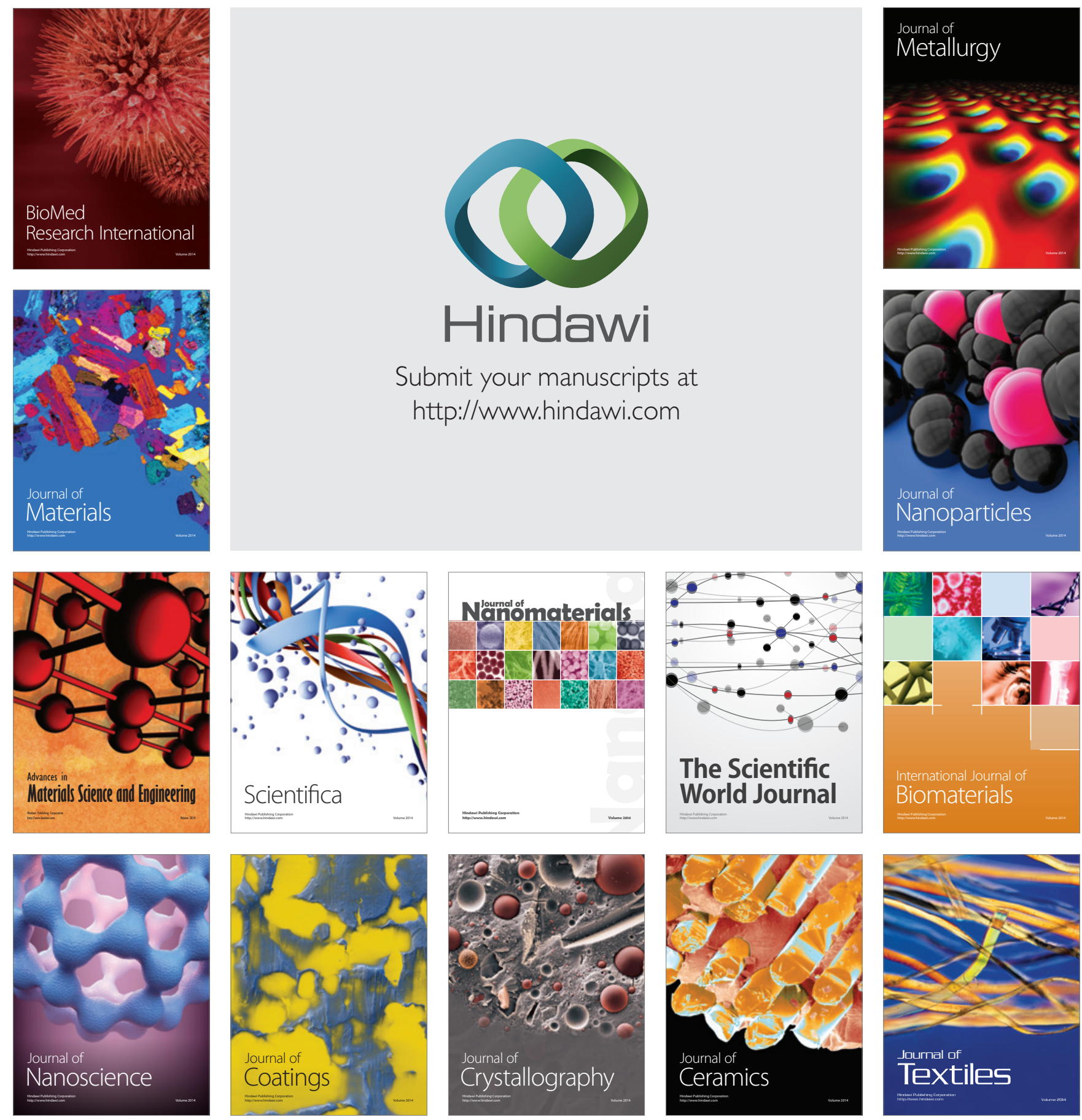